\title{
Educación Superior Comparada: Tendencias Mundiales y de América Latina y Caribe
}

\author{
Francisco López Segrera
}

Resumen: El objetivo de analizar las principales tendencias mundiales y regionales de la educación superior - a partir de las propuestas de las Conferencias Mundiales de educación superior de UNESCO de 1998 y de 2009 y de su consideración como un bien público - es contribuir a promover las transformaciones necesarias de sus tendencias principales. Tales como: la expansión cuantitativa; una privatización creciente; la diversificación institucional; su internacionalización y el crecimiento de las restricciones en el financiamiento público.

Palabras claves: Educación Superior. Tendencias. Acreditación. Rankings. Financiamiento. Internacionalización.

\section{Comparative Higher Education: Worldwide Trends and of Latin America and the Caribbean.}

Abstract : The aim is to analyze the key world and regional trends of higher education - according to the proposals of the World Conferences of Higher Education of UNESCO of 1998 and of 2009 and the consideration of it as a common good - and to help promote the necessary transformations of its principal trends. Such as: the quantitative expansion; an increasing privatization; institutional diversification; its internationalization; and the growth of the restrictions in the public financing.

Key words: Higher Education. Trends. Accreditation. Globalization. Rankings. Financing. Internationalization.

\section{Educação Superior comparada: Tendências mundiais e da América Latina e Caribe}

Resumo: O objetivo é analisar as tendências globais e regionais no ensino superior - a partir das propostas da Conferência Mundial de Educação Superior da UNESCO, em 1998 e 2009 e sua consideração como um bem público - é ajudar a promover as mudanças necessárias em suas principais tendências, tais como: flexibilização quantitativa; crescente privatização; diversificação institucional; internacionalização e crescimento das restrições ao financiamento público.

Palavras-chave: Educação superior. Tendências. Acreditação. Globalização. Rankings, Financiamento. Internacionalização. 


\section{Educación Superior y Globalización}

Los valores tradicionales de la Universidad siguen siendo válidos (la autonomía, la libertad de cátedra, la investigación, el trabajo de los estudiantes, la evaluación), pero son valores amenazados en el contexto de la globalización al cuestionarse el ideal de lo público y del bien común.

Peter Drucker dijo hace una década que en 20 años la universidad sería muy diferente y aún habló de la desaparición posible de las universidades en su forma tradicional. Sin embargo, pese a los grandes a grandes cambios en el mundo y en la enseñanza superior, hay una indudable continuidad en la historia de las universidades a nivel mundial. Según Altbach (2012), la Universidad de 2050 se parecerá en sus aspectos esenciales a la universidad actual, aunque con cambios significativos en muchos aspectos.

El objetivo de analizar estas cuestiones es contribuir a promover las transformaciones necesarias de las tendencias principales de la ES. Tales como: la expansión cuantitativa; una privatización creciente; la diversificación institucional; el crecimiento de las restricciones en el financiamiento público. Con el fin de proporcionar una respuesta adecuada a problemas apremiantes como: la reducción de la inversión pública, las inadecuadas políticas gubernamentales, y la estructura rígida e inflexible de las relaciones con el sector productivo. Las universidades tienen que lidiar con estos problemas a nivel nacional e internacional, sobre todo en los países en vías de desarrollo.

En los documentos de "La Conferencia Mundial sobre la Educación Superior" (1998), convocada por la UNESCO, y en las Comisiones de Seguimiento de dicha Conferencia, se hicieron sugerencias valiosas de cómo afrontar los desafíos más urgentes. Como por ejemplo: la actualización permanente de los profesores, de los contenidos y del currículo; la introducción de redes electrónicas para el aprendizaje; traducción y adaptación de las principales contribuciones científicas; modernización de los sistemas de gestión y dirección; e integración y complementación de la educación pública y privada así como de la educación formal, informal y a distancia.

También en la Declaración Final de la Conferencia Regional de Educación Superior (2008), celebrada en Cartagena como preparatoria de la Conferencia Mundial que se celebraró en UNESCO en julio de 2009, se hicieron planteamientos de especial relevancia, al igual que en él posterior Plan de Acción.

La Conferencia Mundial sobre Educación Superior 2009 (CMES) se celebró en la sede de la UNESCO en París del 5 al 8 de julio de 2009. En ella se analizaron los cambios desarrollados en la educación superior desde la 
primera Conferencia Mundial de 1998 y se centró en "Las Nuevas Dinámicas de la Educación Superior y de la Investigación para el Cambio Social" (LÓPEZ SEGRERA, 2010).

El discurso dominante en la Conferencia fue el papel que podría desempeñar la enseñanza superior para que concluyese la recesión global y qué medidas tomar para que esta crisis no la afectase. Esto fue acompañado por declaraciones sobre la ES como bien público (a diferencia de un servicio público); la necesidad de la equidad en el acceso y de la excelencia; significado de la crisis de valores; la necesidad de inversión sostenida en ES (de los estados nacionales, de las organizaciones internacionales como el Banco Mundial y UNESCO, de organizaciones multilaterales y de otros donantes); sociedades públicas/privadas para el desarrollo de la ES ; libertad de cátedra y autonomía institucional; la necesidad de sistemas de garantía de calidad globalizados, como protección ante los nuevos proveedores ; la necesidad de desarrollar las TIC; de innovación en métodos y contenidos; importancia del desarrollo de redes de investigación y de construcción de capacidades entre países desarrollados y en desarrollo; y posibilidades de empleo para los graduados.

Tras un largo debate a lo largo de la CMES 2009 se incluyó el concepto de la educación superior como bien público en el Comunicado Final y no como servicio público, criterio que defendían otros participantes.

En su condición de bien público - afirma el Comunicado Final- y de imperativo estratégico para todos los niveles de la enseñanza, y por ser fundamento de la investigación, la innovación y la creatividad, la educación superior debe ser responsabilidad de todos los gobiernos y recibir su apoyo económico. Como se destaca en la Declaración Universal de Derechos Humanos, "el acceso a los estudios superiores será igual para todos, en función de los méritos respectivos" (Artículo 26, párrafo 1) (UNESCO, 2009).

\section{Antecedentes Históricos}

En América Latina y el Caribe (ALC) se produjo en el siglo XIX la sustitución de la universidad colonial (modelos de Salamanca y de Alcalá de Henares) por el modelo napoleónico de universidad. No se adoptó el modelo alemán Humboldt de investigación, pero tampoco al adoptar el modelo francés napoleónico que hacía hincapié en la formación de profesionales se crearon los Institutos de Investigación. En ALC se adoptó una versión 
modificada del Modelo Napoleónico. El desarrollo industrial dependiente de América Latina y el Caribe no requería altos grados de innovación científica y tecnológica, pues no se producían bienes de capital. Se compraba a EE.UU. y a los países desarrollados tecnología obsoleta. Esta es una de las razones claves para explicar el nivel de atraso de ALC en su integración a la sociedad del conocimiento. Los países europeos - y luego EE.UU. e incluso Japón - le daban una gran importancia a la investigación-desarrollo, pues competían por un mercado mundial capitalista donde los países con los sistemas productivos más avanzados tecnológicamente eran los que obtenían las mayores ganancias y tenían la hegemonía no solo económica, sino también militar. Sin embargo, el capitalismo dependiente latinoamericano, que derivaba sus mayores ingresos de la exportación de materias primas y no de bienes manufacturados, no priorizó por tanto el desarrollo científico, de ahí nuestro atraso actual.

Somos los universitarios los que debemos liderar el cambio y la innovación desde dentro de nuestras instituciones, porque somos los únicos que las conocemos a fondo. Para ello se requiere una amplia información mundial sobre la universidad, conocimiento acerca de las mejores experiencias de transformación de la universidad, un proyecto institucional sólido, disposiciones normativas flexibles y una decidida voluntad política de cambio. Con estos prerrequisitos, las reformas no serían episodios espasmódicos e inconclusos dado que la universidad se auto-reformaría de forma permanente.

\section{Tendencias mundiales de la Educación Superior}

Las perspectivas de la sociedad del conocimiento y de la innovación estarán condicionadas por las tendencias mundiales de la educación superior.

Pudiéramos resumirlas del modo siguiente:

a) Masificación: la tasa bruta de matrícula (TBM) a nivel mundial pasó de 13 millones de estudiantes en 1960 a 198 millones en 2013 según el último Informe del Instituto de Estadística de UNESCO de junio de 2015. Las diferencias regionales y nacionales en tasas de matrícula revelan la desigualdad mundial: 8\% en África Subsahariana, 77\% en América del Norte y Europa Occidental, 44\% en América Latina y el Caribe, 26\% en Asia Central, 23\% en Asia Occidental y del Sur, 33\% en Asia del Este y Pacífico, 28\% Estados Árabes, 71\% Europa Central y del Este (UNESCO.UIS, 2015). 


\begin{tabular}{|l|c|c|}
\hline PROMEDIOS REGIONALES & $\begin{array}{c}\text { MILLONES DE } \\
\text { ESTUDIANTES }\end{array}$ & $\begin{array}{c}\text { TASA DE } \\
\text { MATRÍCULA }\end{array}$ \\
\hline MUNDO & 198.567 & $33 \%$ \\
\hline ESTADOS ÁRABES & 9.441 & $28 \%$ \\
\hline EUROPA CENTRAL Y DEL ESTE & 20.512 & $71 \%$ \\
\hline ASIA CENTRAL & 2.175 & $26 \%$ \\
\hline ASIA DEL ESTE Y PACÍFICO & 60.665 & $33 \%$ \\
\hline ALC & 23.688 & $44 \%$ \\
\hline $\begin{array}{l}\text { A, DEL NORTE Y EUROPA } \\
\text { OCCIDENTAL }\end{array}$ & 37.669 & $77 \%$ \\
\hline ASIA OCCIDENTAL Y DEL SUR & 37.786 & $23 \%$ \\
\hline ÁFRICA SUB-SAHARIANA & 6.600 & $8 \%$ \\
\hline
\end{tabular}

b) La educación se ha hecho permanente dado al crecimiento exponencial del conocimiento: en el año 2005 las corporaciones de EE.UU. gastaron 15 mil millones de dólares en entrenar a sus empleados.

c) La aparición de las TIC implica que el equipamiento de una universidad es más costoso. La exclusión de ellas implica la segregación del estado del arte de la sociedad del conocimiento.

d) El incremento vertiginoso de la movilidad académica internacional favorece a los alumnos de los países desarrollados y de ciertos países asiáticos y tiende a incrementar la "fuga de cerebros". Los estudiantes internacionales pasaron de 2.5 millones en el 2004 a 4 millones en el 2012 de acuerdo a las últimas estadísticas de UNESCO de 2014. En EE.UU. estudia alrededor de la tercera parte de ellos, y casi el total del resto lo hacen en Europa Occidental, Canadá y Australia. Cerca del 70\% de los estudiantes internacionales van a estos destinos. América Latina y el Caribe (ALC) apenas reciben anualmente el $2 \%$ de los estudiantes internacionales (UNESCO.UIS, 2015).

e) La privatización de la educación superior se incrementa aceleradamente en América del Norte, América Latina y el Caribe, en Asia y en los países del Este de Europa y Rusia. Sólo en las regiones de Europa Occidental y África sigue predominando la educación superior pública financiada casi en su totalidad por el Estado.

f) La crisis de la profesión académica - debido a los bajos salarios entre otros factores - es un fenómeno que se da en forma aguda en los paí- 
ses en desarrollo, pero que también afecta a los profesores del mundo desarrollado.

g) La inequidad en el acceso por motivos de género, étnico, religioso o de clase social, sigue privando a muchos con méritos suficientes para cursar estudios universitarios.

h) El impulso hacia el perfeccionamiento de los procedimientos de gestión, la evaluación, la rendición de cuentas y los procesos de acreditación, muchas veces han tenido un carácter positivo. Sin embargo, en más de una ocasión se han caracterizado por su aspecto burocrático y formal y en otras han servido de pretexto para acelerar procesos de privatización o de reducción de fondos estatales.

i) Hay una carencia de actualización y flexibilidad en el currículo de la mayoría de las IES y en los cursos de postgrado, con la excepción de una minoría de universidades nacionales públicas y unas pocas privadas de calidad.

j) Las IES de educación superior privada son las de más alto nivel (Harvard, ITAM, Externado de Colombia, universidades católicas) y las de más bajo nivel simultáneamente (universidades garaje o patito). Estas últimas se denominan de "absorción de demanda", pues a ellas van a cursar sus estudios todos aquellos que no tienen los requisitos para acceder a las universidades privadas de élite ni a las públicas.

k) Otro fenómeno nuevo es la emergencia de "seudouniversidades", instituciones que no se corresponden con la definición tradicional de la universidad, pero que ofrecen "entrenamiento especializado en una variedad de áreas". La mayoría de ellas son entidades con ánimo de lucro (forprofit) cuya preocupación esencial no son los valores ni la calidad de la docencia, sino obtener ganancias. Como ejemplos podemos poner a la Universidad de Phoenix que pertenece al Grupo Apollo (ALTBACH, 2006, p. 204.)

1) Una de las consecuencias negativas de la globalización económica es la propuesta de la OMC de incluir la educación superior como un servicio sujeto a las regulaciones del GATS. Aunque esta propuesta aún no ha sido aprobada, los países - EE.UU., Nueva Zelanda, Australia, Japón- que obtienen importantes ganancias por concepto de estudiantes extranjeros defienden con fuerza esta iniciativa. 
m) Existe la tendencia de que IES de los países en desarrollo soliciten ser acreditadas por agencias de acreditación de países extranjeros, en especial de EE.UU., sin percatarse que estas agencias desconocen los valores y necesidades de desarrollo autóctono por estar conformadas para otra realidad. Puede ser positivo invitar a expertos a incorporarse a los equipos nacionales de acreditación, pero siempre que estos procesos estén elaborados a partir de valores, normas y necesidades autóctonas.

n) Los rankings están cada vez más de moda. A mediados de los $80 \mathrm{~s}$ el Asian Wall Street Journal incluía, en una lista de las 10 mejores universidades del mundo, sólo 4 (Cambridge, Oxford, Sorbonne, Tokio) fuera de EE.UU. Según Altbach(2008) algunos de estos ranking tienen cierta respetabilidad como el de U.S. News y el del Times HigherEducationSuplement (THES). Se debate mucho acerca de los indicadores que utilizan el THES y la ShangaiJiaoTongUniversity. Lo que ocurre es que los ranking están construidos sobre la base de parámetros - número de Premios Nobel, profesores de la universidad con trabajos en el citationindex, doctorados y maestrías, equipamiento, financiamiento...propios de las universidades de "clase mundial" del mundo anglosajón, en especial de EE. UU. e Inglaterra y que además privilegian las ciencias duras en detrimento de las sociales y humanas.

o) Aplicar las normas y valores de las principales potencias académicas no medirá en forma exacta la calidad a nivel mundial, ni dará lugar a ranking mundiales de interés. En el competitivo y orientado hacia el mercado mundo académico del siglo XXI, los ranking son inevitables y probablemente necesarios. El desafío es asegurar que provean criterios exactos y relevantes y midan las cosas adecuadas (ALTBACH, 2006, p. 80).

p) Es cada vez mayor el debate en torno a las universidades de categoría mundial (World-ClassUniversities), refiriéndose este concepto a las que tienen una excelencia reconocida mundialmente (Yale, Harvard, Oxford, Cambridge, Sorbonne, Tokio...). Sin embargo, los países en desarrollo, en vez de emular para obtener indicadores muchas veces difíciles de alcanzar en sus condiciones específicas, deberían prestar más atención a un ideal de universidad que propulse el desarrollo sostenible de carácter autóctono. Más importante que tener los indicadores de las "worldclassuniversities" o "researchuniversities", más importante que 
tener un premio Nóbel, es garantizar que existan buenas escuelas de medicina y buenos programas de formación de ingenieros agrónomos y educadores para garantizar un nivel adecuado de "capital humano y social", esto es, de recursos humanos en condiciones de generar desarrollo con equidad en las "universidades nacionales".

q) Los procesos de evaluación y acreditación - que nos brindan mayor información sobre las IES, la masificación de la educación superior, su comercialización, el ataque a la concepción de su condición de bien público, la emergencia de las TIC, el auge de los nuevos proveedores y el deterioro de las condiciones salariales y de trabajo de la profesión académica, entre otros factores, - han llevado a un debate sobre lo que muchos consideran un creciente fenómeno de corrupción académica. Es decir, a la obtención de calificaciones, títulos universitarios, puestos académicos o certificaciones de acreditación en el caso de las IES, mediante procedimientos irregulares tales como: pago de sobornos, venta de títulos sin cursar los estudios adecuados, vender una plaza a un candidato en lugar de otorgarla a quién corresponde, acreditar a una IES o programa sin los requisitos a cambio de pagar cierta cantidad.

r) En el 2011 se produjeron intensas movilizaciones estudiantiles en Chile que demandaban una educación superior pública financiada por el Estado. También en Colombia, a partir del 12 de octubre de 2011, se produjeron manifestaciones de los estudiantes docentes, trabajadores universitarios y sindicatos en todo el territorio del País, como protesta por el Proyecto de Reforma a la Educación Superior presentado por el Gobierno Nacional y que luego fue retirado.

s) Las IES están influidas y condicionadas por las políticas del Estado-Nación y por las tendencias globales del sistema-mundo capitalista. Parece ser que estas influencias sobre la Universidad son mucho más poderosas que los cambios y transformaciones que pueden producir las IES dentro de ellas mismas y en la sociedad donde radican. Tal vez por esto se produce una cierta frustración al ver que los impactos de las políticas con ansias transformadoras (Conferencias Mundiales de Educación Superior de UNESCO, 1998; 2009) son de poca intensidad. Esto nos lleva a preguntarnos: ¿Es posible una transformación profunda de la Universidad sin transformar previamente la sociedad? 


\section{Principales tendencias de la Educación Superior en América Latina y el Caribe}

Hasta la década de los 80 s, la ES pública con autonomía institucional y académica, tenía el predominio en la región sobre la educación privada. Al final de los 80s y principios de los 90s, la globalización implicó para ALC la aplicación de las estrategias neoliberales. Esto implicó sustituir la política típica del "Estado de Bienestar", por otras de reducción de financiamiento a los servicios públicos y privatización de ellos. Estas estrategias de mercado tuvieron un impacto en la privatización creciente de la ES y en el deterioro de las universidades públicas, debido a la carencia de financiación apropiada entre otros factores. A pesar de esto, durante los 90 s se produjo una gran expansión de la ES en la región.

Veamos más detalladamente estas tendencias predominantes en la ES en LAC - así como otras - entre 1990 y 2015:

a) Las IES pasaron de 5.438 en 1995 (53,7\% privadas) a 7.514 en el 2002 (69.2\% de ellas privadas). Las universidades propiamente dichas pasaron de 812 en 1995 (60,7\% privadas) a 1.213 en el 2002 (69,2\% privadas) y a 3900 universidades y 10,500 IES no universitarias en 2010. (UNESCO.UIS, 2012; GARCÍA GUADILLA, 2006, p.261; LÓPEZ SEGRERA, 2011a)

b) La matrícula en las IES pasó de 7.405.257 estudiantes en 1995 (38,1\% en IES privadas) a 12.186.260 en el 2002 (47,5 privada). En las universidades propiamente dichas la matrícula pasó de 5.070 .731 en 1995 (30,4\% privada) a 8.316 .649 (40,1 privada) en el 2002. Según las últimas cifras disponibles, la TBM total en LAC era de 23,688,000 en 2013 (UNESCO.UIS, 2015).

c) En el 2003, el promedio de matriculación en la ES en instituciones privadas fue del $50 \%$, alcanzando los porcentajes más altos en países como: Brasil y Chile (70 \%); Colombia: $60 \%$; Centroamérica: aproximadamente el $60 \%$; México: $40 \%$; Argentina: $20 \%$; Uruguay: 10 $\%$; y para Cuba del $0 \%$. (UNESCO- IESALC, 2006; FERNÁNDEZ LAMARRA, 2008).

d) Hay megasistemas de educación superior con más de cuatro millones de estudiantes (Brasil: 6,553,000); sistemas grandes con más de dos millones - México $(2,847,000)$ y Argentina $(2,387,000)$; medianos 
-entre un millón doscientos mil y 500,000 estudiantes- como son los casos de Chile (876,000), Colombia $(1,674,000)$, Perú $(1,207,000)$ y Venezuela $(2,123,000)$; pequeños - entre 500,000 y 150,000 estudiantes- en países como Bolivia (346,056), Cuba (801,000), Ecuador (535,000), Guatemala (114,764), y República Dominicana $(293,565)$ ; y muy pequeños - entre 500,000 y 150,000- como es el caso de Costa Rica (110,117), El Salvador (150,000), Honduras (148,000), Nicaragua (103,577), Panamá (135,000), Paraguay (236,000) y Uruguay $(161,000)$. Los sistemas de ES en todo el Caribe no español - anglófono, francófono, etc. -son también muy pequeños: Jamaica (71,000), Trinidad Tobago (16,920), Santa Lucía $(2,197)$ (UNESCO. UIS, 2014, Tabla b).

\begin{tabular}{|l|l|c|}
\hline REGIÓN & PAís & TBM/GER \\
\hline AMÉRICA LATINA Y EL CARIBE & Argentina & $80 \%$ \\
\hline & Venezuela & $78 \%$ \\
\hline & Chile & $79 \%$ \\
\hline & Uruguay & $63 \%$ \\
\hline & Cuba & $48 \%$ \\
\hline & Colombia & $48 \%$ \\
\hline & México & $30 \%$ \\
\hline & Jamaica & $29 \%$ \\
\hline & Honduras & $21 \%$ \\
\hline
\end{tabular}

e) La tasa de matrícula (TBM) varía de países con una tasa del $60 \%$ o más (Argentina 80\%, Venezuela, 78\%, Chile 79\%; a países con tasas entre 30 y $60 \%$ : Cuba, 48\%, Colombia, $48 \%$ y a países con menos del 31\%: México 30\%; Jamaica, 29\%; Honduras 21\%. La tendencia es al aumento en el \% de estudiantes universitarios matriculados, pero en algunos países - Saint Lucia, Trinidad and Tobago- se ha producido una ligera disminución (UNESCO.UIS, 2015).

f) La tasa bruta de matrícula promedio ha pasado de $17 \%$ a principios de los 90s a 44\% en la actualidad (2012) (UNESCO.UIS, 2015).

g) El gasto promedio por estudiante (USA \$2380) es mucho menor al de los países desarrollados. Además, mientras en América Latina en una 
muestra de 12 países los gastos gubernamentales promedio en educación varían entre el 2,5 y el 3,5\% del PIB en los países de la OCDE (2008) este gasto promedio es del $4,5 \%$.

h) El gasto privado en educación superior financia alrededor del 50\% de la matrícula total.

i) En el pregrado no se pagan aranceles de matrícula como norma - salvo en el caso de algunos países como Chile -, pero en el postgrado sí se pagan, pues la mayor parte de los que ofrece la educación superior pública no son gratuitos.

j) El $60 \%$ de la matrícula total regional de ES está concentrada en tres países: Brasil, México y Argentina. La educación superior pública ha tenido gran relevancia en el apoyo a los postgrados y en el desarrollo de la investigación científica. México y Brasil tienen una matrícula de 100,000 estudiantes en cursos de postgrado (GAZZOLA, 2008).

k) La población de bajos recursos tiende a resultar excluida de la educación superior pública por haber asistido a escuelas de menor calidad, lo que les impide aprobar los exámenes de selección. Muchos van a ingresar en las universidades de menor calidad del sector privado.

La tasa de matrícula en ALC pasó del $17 \%$ al principio de los años 90 al $44 \%$ en 2013 de acuerdo a las últimas estadísticas de UNESCO de 2015, pero esto no implicó, por lo general, una disminución proporcional de la desigualdad. Sin embargo, en algunos países como Cuba, Argentina, Brasil, República Dominicana, y Venezuela, la expansión de la tasa de matrícula implicó una disminución de la desigualdad social, pese a que en algunos de estos países, como Brasil y República Dominicana, el índice de pobreza es todavía alto.

El problema en ALC es que un alto índice de pobreza y un bajo acceso a la educación básica en muchos países tuvo como resultado que las clases medias fueron casi las únicas beneficiadas de este crecimiento de la tasa de matrícula Sólo el $23 \%$ (mujeres) y el $26 \%$ (los hombres) del quintil más pobre tiene éxito en el completar la enseñanza secundaria en ALC, pero en el quintil más rico las cifras son del 81 y el $86 \%$. En los países Andinos, se estima que apenas el $45 \%$ de la población que completa la enseñanza secundaria tiene acceso a la enseñanza superior. En estos países el acceso a la ES para la población entre 20-24 años en las áreas urbanas que pertenecen al quintil más pobre, es menos del $3 \%$. 
Los países latinoamericanos tienen el promedio más bajo en el "Índice de Equidad de Educación" a nivel mundial. En la mayor parte de los países latinoamericanos, alrededor del $50 \%$ del quintil más rico tiene acceso a la enseñanza superior. En el quintil más pobre esta proporción está entre el $10 \%$ y el $20 \%$. Los grupos de bajos ingresos de la población tienden a ser excluidos de las instituciones de educación superior. Estos grupos, reciben una educación básica de menor calidad, que les impide ser seleccionados para ingresar en la educación terciaria y en especial en instituciones de calidad.

Chile y Brasil tienen los sistemas de educación superior más elitistas. A pesar de la política de acción afirmativa desarrollada por el Gobierno de Lula, la TBM para brasileños negros entre 19 y 24 años es del $6 \%$, comparado al $19 \%$ para brasileños blancos. En Brasil, el $59 \%$ de los estudiantes matriculados en universidades públicas y el $74 \%$ de los que estudian en las privadas pertenecen al quintil de ingreso más rico y esta proporción es el $52 \%$ en Chile. En México, la proporción es del $58 \%$ de los estudiantes del quintil más rico y el $6 \%$ del más pobre. En Chile de 65 y 8 . En Ecuador de 42 y 6 . En Cuba y Argentina es donde se observan las menores desigualdades y TBM más elevadas. Otro serio problema en ALC es la deserción académica que afecta en especial a los estudiantes del quintil inferior, es decir, a los más pobres.

Para concluir podemos señalar que los países que registran las mejores TBM en la enseñanza superior son aquellos con: los mejores niveles de desarrollo humano, inversiones públicas elevadas en educación, los niveles más bajos de pobreza, altos niveles de participación en una educación básica de calidad y políticas públicas dirigidas a aumentar el acceso a la educación básica y superior y a disminuir la inequidad.

1) El modelo de financiamiento que sigue predominando en la educación superior pública es el histórico negociado y en la privada el pago de matrícula. Sin embargo, recientemente se ha introducido en las universidades públicas de algunos países (México, Chile, Argentina) el presupuesto por incentivos y las fórmulas de financiamiento para reorganizar la asignación y el uso de fondos, vinculando recursos y salarios a resultados.

m) Los gobiernos de algunos países (Uruguay) han introducido fondos solidarios para dar más posibilidades a los alumnos de estratos sociales desfavorecidos, pero con méritos académicos. 
n) La región ha atraído a varias instituciones de educación superior extranjeras, con y sin ánimo de lucro, incluyendo la educación superior transfronteriza Se observa el aumento rápido de las Instituciones Extranjeras de ES (procedentes de EE.UU., Europa y de otros países de la región) con modalidades diversas: educación de distancia (102), unidades descentralizadas de la sede (50), alianzas (125) y acuerdos (816). En muchos casos estas IES no tienen la calidad apropiada o violan regulaciones nacionales, muy escasas en lo que a la ES transnacional se refiere. (GARCÍA GUADILLA, 2006; DIDOU, 2005).

o) La internacionalización de la ES está presente en ALC a través de Programas intercontinentales como ALFA Y COLOMBUS; subregionales, como la Asociación de Universidades del Grupo de Montevideo (AUGM), para MERCOSUR; por redes de Iberoamericanas (RIACES) agencias regionales (IESALC) y redes de universidades católicas (FIUC, AUSJAL). También la internacionalización se expresa a través de varios programas de diversos países, y redes de universidades regionales y mundiales, que otorgan financiamientos y becas a profesores y estudiantes de ALC. Se pudiera catalogar a este tipo de internacionalización como sin ánimo de lucro - a diferencia de la que ofrecen los nuevos proveedores-, pero hay voces que señalan que detrás de algunas de estas estrategias de becas y financiamientos está el objetivo de sustraer a la región muchos de sus más valiosos profesores e investigadores, así como alumnos con perspectivas. (DIDOU, 2005, p. 133; CINDA, 2007).

p) De acuerdo con UNESCO, ALC recibió en 2012 62,468 estudiantes extranjeros, de un total mundial de 4,009,312 millones, mientras que América del Norte y Europa Occidental recibieron un total de 2,279,068. Esto indica que los países de la región no constituyen destinos atractivos para los estudiantes internacionales. Información que ratifica este hecho, es que ninguno de los países de América Latina hoy en día aparece en la lista de los veintitrés destinos que atraen mayor número de estudiantes extranjeros. (UNESCO.UIS, Tabla b, 2014 ).

q) En 14 países 31 instituciones otorgan créditos educativos por un monto de USA 400 millones. La Caixa Económica Federal de Brasil cubre el 50\% de este monto y le siguen el ICETEX de Colombia y FUNDAYACUCHO de Venezuela. También Cuba otorga miles de becas 
a estudiantes de la región y financia una Escuela Latinoamericana de Medicina.

r) La privatización de la ES en América Latina y el Caribe llevó a la diversificación de las IES y, en muchos casos, a la falta de calidad. A partir de la década de los 90s casi todos los países de la región crearon organismos de acreditación. La Comisión Nacional para la Evaluación de la Educación Superior y el Consejo para la Acreditación de la Educación Superior (México); el Consejo Superior de Educación (Chile); el Consejo Nacional de Acreditación (Colombia); la Comisión Nacional de Evaluación y Acreditación Universitaria (Argentina); el Sistema Centroamericano de Evaluación y Acreditación de la Educación Superior (Centroamérica); CAPES (Brasil); el Mecanismo Experimental de Acreditación de Carreras para el Reconocimiento de Títulos de Grado Universitario (MEXA/MERCOSUR) (DIAS SOBRINHO, 2005).

Los procesos de acreditación deberían trascender su visión de la calidad como algo meramente técnico, e incluir en los indicadores para la validación oficial y formal de una universidad o de sus programas, entre otros aspectos, indicadores de compromiso ético y social. Estos indicadores son:

a) El grado de relevancia (de acuerdo con las necesidades sociales) del proyecto institucional de las universidades y de los programas en cuanto a las distintas carreras profesionales.

b) La investigación universitaria debería estar enfocada hacia problemas globales como: la paz, el desarrollo sostenible, la pobreza y la diversidad cultural. También debería contribuir a generar conocimiento en áreas clave para el desarrollo del país. Las universidades deberían transferir estos conocimientos para satisfacer las necesidades locales de la sociedad.

c) Los planes de estudio deberían adaptarse a los cambios locales y nacionales del mercado laboral.

d) La buena gestión, la transparencia y la rendición de cuentas en procesos como la evaluación y la acreditación.

e) La calidad de la enseñanza y la investigación por parte del 
profesorado, medidas según los valores universales y según los valores específicos de los ciudadanos y el compromiso social.

f) La calidad de la integración de los estudiantes en la sociedad y el mercado laboral y la forma de actuar de acuerdo, o no, con los valores del compromiso social.

g) Los indicadores de la acreditación deberían garantizar un equilibrio adecuado entre la autonomía y los procedimientos de garantía de la calidad.

h) El grado de coordinación de los gobiernos y las organizaciones con las normas internacionales de acreditación y garantía de la calidad, como las desarrolladas por la UNESCO.

Adicionalmente, los procesos de acreditación y garantía de la calidad deberían incluir indicadores como los siguientes: a) a que quintiles de la población pertenecen sus alumnos y graduados; b) analizar si existen programas de becas para permitir el acceso a la universidad a los estudiantes de estratos económicos desfavorecidos, que teniendo los méritos no acceden a la universidad por razones económicas; c) valorar si la universidad da seguimiento a sus graduados y que porcentaje de ellos trabajan en lo que estudiaron y con qué remuneración; d) habría que analizar los acuerdos entre la universidad y las empresas para ver si están acordes con los valores de responsabilidad social que debe cumplir la universidad; e) se tendría que valorar si existen cursos en los grandes temas de nuestro tiempo tales como: problemas ambientales, objetivos del milenio, solidaridad internacional, pobreza, hambre, energía nuclear y SIDA; f) debía considerarse si en los programas de investigación y extensión se incluyen proyectos de colaboración Norte-Sur o bien Sur-Sur, con el objetivo de que las universidades que tienen más recursos hagan aportes a las que menos recursos tienen; g) un indicador para otorgar la debida acreditación y certificación a instituciones y programa sería evaluar en qué grado contribuyen a la solución de problemas importantes para la comunidad con una visión de desarrollo sostenible y en el caso de los países en desarrollo para su desarrollo sostenible autóctono.

i) Hay una tendencia regional a la institucionalización jurídica de los sistemas de educación de superior a través de una ley 
general de educación -también denominada ley marco- y de una ley específica de educación superior. Sin embargo, muy pocos países (Chile) tienen una ley que regule los procesos de acreditación.

j) De especial interés es el Proyecto $6 \times 4$ que se ha gestado en el CENEVAL (México) en colaboración con el Programa Columbus. Es un ensayo piloto que tiene el objetivo de construir un espacio latinoamericano común de educación superior tomando en cuenta la experiencia europea de Bologna. (DIAS SOBRINHO, 2006, p. 219).

k) La Universidad de las Antillas (UWI, en sus siglas en inglés) es la institución principal de educación superior en el Caribe Anglófono. Tiene campus en tres países: Jamaica, donde se encuentra el mayor de ellos, al que le siguen el de Trinidad y Tobago y el de Barbados. Acoge a unos 19.600 estudiantes de 17 países de la zona. Si se añaden las inscripciones a distancia, la matriculación total asciende a 23.200 alumnos. El índice de matriculación difiere de un país a otro: el 31\% en Jamaica y el $12 \%$, en Trinidad y Tobago. Según la definición de Martin Trow (1974), en el Caribe de habla inglesa la educación superior en algunos países como Jamaica está «masificada», mientras que en otros, como Trinidad y Tobago, es «elitista», pero nunca es universal, salvo en las Islas Vírgenes. (LÓPEZ SEGRERA et al., 2006;2008; 2009; UNESCO, 2007; 2008; 2009. Ver en la Web del IESALC https://www.google. es/\#q=IESALC+UNESCO+2006 los diversos estudios sobre la educación superior en el Caribe).

1) El presupuesto promedio de la ES en ALC es inferior al 1,5\% del PIB, lo que es claramente insuficiente para satisfacer las necesidades de un sistema en desarrollo y en particular para atender a las demandas nacionales para la investigación.

m) El promedio de la inversión en Ciencia y Tecnología de los países de la Región es del 0,72\% del PIB. El resultado, entre 
otros, como se puede observar en los Anexos 1, 2 y 3, es la deficiente situación de la región en lo que se refiere a Investigación \& Desarrollo, producción académica y producción científica ${ }^{1}$.

n) Las CMES de UNESCO de 1998 y 2009 y sus Reuniones de Seguimiento, tuvieron como impacto central el ofrecer un marco teórico consensuado para transformar la ES en ALC. A nivel macro, esto implicó una visión general compartida acerca de cómo conducir los procesos de transformación de las IES de carácter público - e incluso privado - mediante la evaluación institucional, con el objetivo de perfeccionar los sistemas de gestión y financiamiento, y privilegiar políticas encaminadas al logro de la equidad ampliando el acceso. Pero también implicó mucho más, esto es, que la Universidad debía ser un agente clave de transformación de la sociedad construyendo alternativas viables en medio de la complejidad y la incertidumbre y en una coyuntura signada por la crisis de paradigmas y alternativas. La cooperación internacional y la construcción de redes ha sido uno de los mayores aportes de las CMES en la región, así como la innovación y la internacionalización de la ES. IESALC-UNESCO ha desempeñado un papel de liderazgo en este sentido, como una alternativa a la visión del Banco Mundial en la década de los 90s. EL Banco Mundial consideró que solo debía invertirse en la educación básica y no en la superior y estimuló la privatización de la educación superior como solución a la creciente demanda. El liderazgo del IESALC-UNESCO se hizo evidente, una vez más, en la CRES 2008 celebrada en junio de ese año en Cartagena, Colombia. También el liderazgo latinoamericano en la defensa de la educación superior como un bien público fue evidente en la Conferencia Mundial de Educación Superior celebrada en UNESCO, Paris en 2009.

1 Ver en la web del IESALC, la ponencia que presentó F. Chaparro a la CRES 2008. También en los trabajos de J. J. Brunner (2006), publicados en su web citada al final, hay información relevante al respecto. Ver también H. Vessuri (2008). 


\section{Conclusiones}

Algunos de los principales retos que enfrenta la ES hoy en América Latina y el Caribe son: el rápido aumento de la privatización, la insuficiente financiación pública, la falta de acceso de los más pobres, la débil inversión en $\mathrm{I}+\mathrm{D}+\mathrm{i}$, la escasa producción científica, la alta tasa de deserción académica y la baja tasa de matrícula, a pesar de su mejora, en comparación con los países desarrollados.

Somos testigos de la crisis de la universidad en América Latina y el Caribe- $\mathrm{y}$ a nivel mundial -, no sólo en cuanto a la gestión, financiación, acreditación y currículo, sino también en cuanto a su misma concepción. Debemos adaptar a la universidad a un entorno que muestra cambios radicales en los conceptos básicos y en las identidades. Algunos consideran que la falta de socialización está relacionada con cambios radicales en la capacidad de educación y socialización de instituciones tradicionales como la familia y la escuela. El desafío consiste en la construcción de la nueva universidad, en reinventarla en este clima de incertidumbre y crisis económica global, impidiendo el triunfo de pesimismo.

La universidad debe cumplir la función social que espera la sociedad de ella, no sólo en equidad en el acceso, sino también en lo que se refiere a estar en el estado del arte en conocimientos, informaciones y propuestas de soluciones para un adecuado desarrollo sostenible humano y social.

Es el momento de reconocer que, a pesar de avances y de logros en unas pocas IES de excepción, aún en ALC no hemos sido capaces de alcanzar la cristalización de un nuevo modelo de universidad, en el cual la producción de conocimiento predomine, y no su mera transmisión. Una prioridad en este proceso que deberíamos desarrollar, sería la socialización del conocimiento. De este modo, las IES no se limitarían a ser meras instituciones académicas, pues las universidades se convertirían en las líderes del cambio social.

Somos conscientes de que no hay un modelo único de universidad. Hay muchos ejemplos acertados de reformas de universidad y transformaciones que, mediante la adaptación apropiada a las condiciones de ALC, podemos generalizar y aplicar en nuestras universidades ${ }^{2}$.

Pese a que lo expresado más arriba acerca de la ES en ALC pudiera tener una lectura pesimista, las transformaciones recientes que se han producido en la región con la emergencia de gobiernos con hondas preocupaciones sociales, pudiera brindar una coyuntura propicia para la transformación de la educación superior en la dirección de la Agenda de UNESCO y aún de propuestas más radicales. Sin embargo, en muchas de estas experiencias de gobiernos con políticas pos-neoliberales que se presentan como alternativa

2 La metodología aplicada en los estudios de "benchmarking" puede ser relevante al respecto. 
al modelo neoliberal, no siempre hay una política educacional que implique una ruptura con políticas tradicionales de educación superior, y mucho menos planes de acción que traduzcan la voluntad política de cambio en una alternativa diferenciada.

\section{Referencia}

ALTBACH, P. Franchising: the mcdonaldization of higher education. International Higher Education, CIHE, Boston, n. 66, 2012.

ALTBACH, P. Reflections on Policy and Practice.International Higher Education,CIHE, Boston, 2006.

ALTBACH, P. The 'Subprime' Market and International Higher Education. International Higher Education, CIHE, Boston,n. 51, 2008.

BRUNNER, J. J. Documentos varios elaborados para el Curso impartido en el Centro Universitario Salesiano de Sao Paulo, 2006. Diponibleen: $<$ http:// mt.educarchile.cl>.

Centro Interuniversitário de Desarrollo - CINDA. Educación superior en Iberoamérica. Informe 2007. Santiago de Chile, 2007.

Conferencia Regional de Educación Superior - CRES 2008. Cartagena, Colombia, 2008.

DIAS SOBRINHO, J. Dilemas da educação superior no mundo globalizado. São Paulo: Casa do Psicólogo, 2005.

DIDOU, S. Internacionalización y proveedores externos de educación superior en América Latina y el Caribe. México D.F.:ANUIES, 2005.

FERNÁNDEZ LAMARRA, N. Una perspectiva comparada de la educación superior en América Latina. Ponencia presentada a Universidad 2008. Habana, 2008.

GARCÍA GUADILLA, C. Financiamiento de la educación superior en América Latina. En: La educación Superior en el Mundo 2006: el financiamiento de las universidades. Madrid: GUNI; Ediciones Mundiprensa, 2006.

GAZZOLA, A. L. Integración académica e internacionalización de la educación superior. Conferencia enUniversidad 2008. Habana, 2008.

LÓPEZ SEGRERA, F. Access and Equity in Latin American and Caribbean Higher Education. IAU, Horizons, v. 17, n. 2, oct.2011. 
LÓPEZ SEGRERA, F. Políticas públicas y Gestao. Vision of the World Conference on Higher Education (WCHE, 2009). Acta Scientiarum. Education, Maringá, v. 32, n. 1, 2010.

LÓPEZ SEGRERA, F.; BROCK, C.; DIAS SOBRINHO, J. Higher Education in Latin America and the Caribbean. IESALC, Caracas, 2009. LÓPEZ SEGRERA, F.; BROCK, C.;DIAS SOBRINHO, J. La educación superior en América Latina y el Caribe 2008. Avaliação, Campinas; Sorocaba,v. 13, n. 2, jul.2008.

Organização para a Cooperação e Desenvolvimento Económico - OCDE: La Enseñanza superior en el 2030. Paris, 2008.

TROW, M. Problems in the Transition from Elite to Mass Higher Education. En: OECD. Policies for Higher Education. General Report of the Conference on Future Structure of Post-Secondary Education. Paris, OECD, 1974.

UNESCO. Conferencia mundial sobre la educación superior - 2009:1 a nueva dinámica de la educación superior y lainvestigación para el cambio social y eldesarrollo. Paris: UNESCO, 2009. ED.2009/CONF.402/2

UNESCO.Global Education Digest. UIS. Montreal, 2012.

UNESCO.UIS. Tertiary Education Statistics. June, 2015.

UNESCO.UIS. Tertiary Education Statistics.March.Montreal, 2014.

UNESCO-IESALC. Informe sobre la educación superior en América Latina y el Caribe. Caracas, 2006.

VESSURI, H. La ciencia y la educación superior en el proceso de internacionalización. Elementos de un marco conceptual para América Latina. UNESCO Forum Occasional Paper Series. Paper no. 3/5. Paris,2008.

Francisco López Segrera - Instituto Internacional de Educación Superior de UNESCO. Tarragona España. Contato: francisco.lopez-segrera@upc.edu

Artigo recebido em 10 de agosto de 2015 e aprovado em 29 de outubro de 2015. 